\title{
DISCOVERY OF SPECTROSCOPIC VARIATIONS IN THE DAB WHITE DWARF GD 323
}

\author{
C. Pereira, P. Bergeron, and F. Wesemael \\ Département de Physique, Université de Montréal, C.P. 6128, Succ. Centre-Ville, \\ Montréal, Québec, Canada, H3C 3J\%. \\ pereira@astro.umontreal.ca, bergeron@astro.umontreal.ca, \\ wesemael@astro.umontreal.ca
}

\begin{abstract}
We report the discovery of spectroscopic variations in GD 323, the prototypical DAB white dwarf. Simultaneous optical spectroscopic observations over five consecutive nights of GD 323 and of PG 1234+482, a non-variable comparison DA white dwarf of similar brightness, are used to reveal quasi-periodic variations in both the hydrogen and helium absorption lines over a timescale of hours. The amplitude of the variation of the equivalent width of $\mathrm{H} \beta$ is $\sim 30 \%$. Moreover, the strength of the hydrogen lines is shown to vary in opposite phase from that of He I $\lambda 4471$. These results suggest that the model currently thought to be the most viable to account for the simultaneous presence of hydrogen and helium lines in GD 323, namely a static stratified atmosphere, may need to be reexamined. Instead, a model with an inhomogeneous surface composition, resulting perhaps from the dilution of a thin hydrogen atmosphere with the underlying helium convection zone, may be a better representation of GD 323. The observed variation timescale of $\sim 3.5$ hours is consistent with the slow rotation rate of white dwarf stars.
\end{abstract}

Subject headings: stars: individual (GD 323) - stars: variables: other - white dwarfs

\section{INTRODUCTION}

The DAB stars represent a class of white dwarfs with hybrid spectra, as weak neutral helium lines are superposed onto the classical hydrogen-line spectrum of DA stars (see, e.g., Wesemael et al. 1993). The prototype of this class, GD 323 (WD $1302+597 ; V=14.52$ ), 
was discovered independently by Oke et al. (1984) and Liebert et al. (1984). The latter made the first attempts at modeling the unusual hybrid spectrum of GD 323, and their results have since been updated and considerably upgraded by Koester et al. (1994) on the basis of a set of high-quality optical spectra of that star. Koester et al. (1994) were led to conclude that a stratified atmospheric structure, consisting of a thin $\left(\log M_{\mathrm{H}} / M_{\star}=-16.8\right)$ hydrogen layer floating on top of a helium envelope, remained the "most promising" explanation of the bulk of the properties of GD 323.

Since this initial discovery, several additional stars spectroscopically classified as DAB stars have been unearthed: G104-27 (Holberg et al. 1990), HS 0209+0832 (Jordan et al. 1993), MCT 0128-3846 and MCT 0453-2933 (Wesemael et al. 1994), PG 1115+166 (Burleigh et al. 2001; Bergeron \& Liebert 2002) and, most recently, PG 1603+432 (Vennes et al. 2004). The sample of DAB stars is quite inhomogeneous, however, as several distinct mechanisms have been called on to account for individual objects: thus, GD 323 could be a stratified object, while it has been argued in the discovery papers cited above that MCT 0128-3846, MCT 0453-2933, and PG 1115+166 are unresolved DA+DB binaries. This has now been confirmed for PG 1115+166 (Maxted et al. 2002) and MCT 0453-2933 (Napiwotzki et al. 2005). Furthermore, HS 0209+0832, while initially thought to be characterized by a homogeneous atmosphere (Heber et al. 1997a), now appears to be undergoing accretion (Wolff et al. 2000). The last object added to the list, PG $1603+432$, seems characterized by a homogeneous composition.

Among those stars, the prototype GD 323 is perhaps the most thoroughly studied object. In their detailed analysis, Koester et al. (1994) investigate four distinct models: homogeneous and stratified composition, unresolved binary and spotted star. Despite this variety of options, they conclude that none of these models provides a completely satisfactory fit to the data, but that the stratified model is the most acceptable match. Given this ambiguous outcome, further investigations of these models appear in order.

An important ingredient in defining the correct model for GD 323 could be the question of variability. While the white light photometry of Robinson \& Winget (1983) excludes photometric variations at the $0.25 \%$ level in the range $10-1200 \mathrm{~s}$, it was suggested by Liebert et al. (1984) that the object be monitored for spectroscopic variations. This need became more acute with the report by Kidder et al. (1992) that the weak He I lines, initially observed by Holberg et al. (1990) in data at high S/N on the DAB star G104-27, were not seen in later spectra of comparable quality. A similar situation is encountered in HS 0209+0832, in which Heber et al. (1997a) report changes in the strength of the neutral and ionized helium lines over a one-year period. In GD 323, earlier spectra obtained by Liebert et al. (1984) and by J. L. Greenstein — initially displayed by Koester (1991a,b) and Wesemael et al. (1993) 
- unfortunately cannot provide any firm conclusion about the variability of that object, as these were obtained with different instrumental setups and spectroscopic resolutions.

To investigate whether GD 323 is a spectroscopic variable, Koester et al. (1994) obtained a series of nine high signal-to-noise ratio, $\sim 7 \AA$ resolution spectra of that object, all secured with an essentially identical instrumental setup. Six of these spectra were obtained within a single night, while the other three had been secured, respectively 15 days, 9 months, and one year earlier. On the basis of these spectra, the best and most homogeneous set secured at that time for a DAB star, Koester et al. (1994) concluded that the available data were compatible with the assumption that the star showed no variability. However, it was our feeling at the time that, while it was clear that there were no large-amplitude changes in the line spectrum of GD 323, the signal-to-noise ratio of the individual spectra was good enough to permit a much more detailed analysis of the variability of GD 323 than had been carried out by Koester et al. (1994). This was undertaken independently by Wesemael et al. (1995), but their analysis could not quite meet the burden of proof associated with an investigation of this type: while their results suggested the presence of small spectroscopic variations in the Koester et al. (1994) data, their analysis also showed that a well-planned observing strategy and a rigorous data reduction process would both be required in order to build a stronger case for the presence, or absence, of low-level spectroscopic variations in GD 323.

Recently, the opportunity arose to revisit the issue of spectroscopic variability of GD 323 , and we succeeded in securing new data for that object that sheds some light on this 20 year-old problem. This paper summarizes the results of this revamped investigation.

\section{OBSERVATIONS}

\subsection{Data Acquisition}

As discussed above, the study of Koester et al. (1994) reveals that the spectroscopic variations in GD 323, if any, are quite small. Hence a careful observing strategy had to be developed to ensure that any variability detected in a series of time-resolved spectroscopic observations is intrinsic to the star and not the result of changes in the atmospheric transparency or of an artifice introduced in the data reduction. The best way to assess the variability of GD 323 is to secure in parallel spectroscopic observations of a constant comparison star. Ideally, one would like to have both GD 323 and the comparison star on the slit in order to obtain simultaneous time-resolved spectroscopic observations. Unfortunately, no such star could be found in the vicinity of GD 323, and we had to rely on a different 
strategy.

We thus selected a comparison star based on the following set of criteria: firstly, we required a star with no known variations. We also required an object whose magnitude was similar to that of GD $323(V=14.52)$. Furthermore, we demanded that our comparison object have few spectral lines and a spectral energy distribution comparable to that of GD 323. Finally, we wanted an object that was as close as possible to our target object in the sky. Examination of the Catalog of Spectroscopically Identified White Dwarfs of McCook \& Sion (1999) revealed that the DA star PG $1234+482$ was the most judicious choice. Past observations of PG $1234+482$ have shown no evidence for variability, it has a visual magnitude of $V=14.42$, and with an effective temperature of $T_{\text {eff }}=55,040 \mathrm{~K}$ (Liebert et al. 2005, hereafter LBH), its spectrum consists of a well-defined continuum with only weak hydrogen lines. Finally, PG $1234+482$ and GD 323 are separated by approximately 15 degrees on the sky.

Since we did not know a priori over what timescales variations might occur, we carried out spectroscopic observations over five consecutive nights, from 2004 February 10 to 14. Both GD 323 and PG 1234+482 were monitored for over 5 hours on the first night, and for about an hour on the remaining nights. These optical spectra were secured using the Steward Observatory $2.3 \mathrm{~m}$ reflector telescope equipped with the Boller \& Chivens spectrograph and a Loral CCD detector. A 4.5 arcsec slit and a 600 line $\mathrm{mm}^{-1}$ grating in first order provided a spectral coverage of 3200-5300 $\AA$ at a resolution of $\sim 6 \AA$ FWHM. Each 600 s spectroscopic observation of GD 323 was immediately followed by a 600 s exposure of PG $1234+482$. The average signal-to-noise ratio per pixel and per exposure is $\sim 85$ for GD 323 and $\sim 100$ for PG 1234+482. In total, 27 spectra of GD 323 and 25 spectra of PG 1234+482 were secured, the vast majority of them under excellent observing conditions.

\subsection{Data Reduction}

The optical spectra were extracted and wavelength-calibrated using the Image Reduction and Analysis Facility (IRAF) standard package. A first cut at the flux calibration was obtained with IRAF using the various flux standards secured during the observing nights. However, in the course of our analysis, we realized that we could take advantage of our multiple spectroscopic observations of PG $1234+482$, and use this star instead as a flux standard, at least in a relative sense. This method is analogous to that used for taking highspeed photometric observations of variable stars, where a constant comparison star is used

to correct the light curve of the target star. The detailed procedure used in the reduction of our spectroscopic observations thus proceeded as follows. 
The first step was to combine our 25 individual spectra of PG 1234+482, flux calibrated with IRAF as described above. This combined spectrum, characterized by a signal-to-noise ratio of $\sim 340$, was then fitted with a grid of synthetic white dwarf spectra, from which we derived $T_{\text {eff }}=54,200 \mathrm{~K}$ and $\log g=7.77$. This effective temperature is within 1.5 $\%$ from that derived by LBH on the basis of a lower signal-to-noise ratio spectrum. A synthetic spectrum calculated with these atmospheric parameters, which matches extremely well the combined observed spectrum, now serves as a noiseless template for the rest of the analysis. Flux calibration functions were then obtained by simply dividing this template by the individual unfluxed spectra of PG $1234+482$ and smoothing the result with a 9-point filter. The application of these smoothed flux calibration functions to the unfluxed spectra of PG 1234+482 ensures that each resulting spectrum matches the synthetic template perfectly. The fluxed spectra for PG 1234+482 are shown in the right panel of Figure 1 for the first night, and in the right panel of Figure 2 for the remaining nights.

The next step was to use these derived functions to flux-calibrate the spectroscopic observations of GD 323. Specifically, we calibrate each spectrum of GD 323 with the function derived from the observation of PG 1234+482 that immediately follows. The resulting fluxcalibrated spectra of GD 323 are displayed in the left panels of Figures 1 and 2. There are two main advantages to our approach: first we obtain flux calibrated spectra of our target star that are as independent as possible of the flux calibration provided by IRAF. Here, the IRAF package is used only to generate the average spectrum of PG 1234+482 used to define the noiseless synthetic template of that star. Once this template is defined, the uncoupling from IRAF in the procedure that generates calibrated spectra of GD 323 is complete (IRAF spectra are used to generate our error estimate in $\S 3$ ). This is a substantial advantage, given that the flux calibration is recognized to be the most delicate step of the reduction procedure. In addition, because of the nearly simultaneous observations of GD 323 and PG 1234+482, our method allows us to monitor small temporal changes in the atmospheric transparency.

As will be discussed further below, our conclusions about the variability of GD 323 do not hinge on the flux calibration procedure developed here, although the quality of the results has certainly benefited from this approach. One should also note that we did not worry about calibrating our spectra in terms of absolute fluxes, as we are mainly interested here in relative flux variations. 


\section{A SEARCH FOR SPECTROSCOPIC VARIATIONS IN GD 323}

\subsection{Single-Night Variations in Equivalent Widths}

With our calibrated spectra in hand, we search for spectroscopic variations by measuring the equivalent widths of the most important hydrogen and helium absorption lines. The most delicate step in this procedure is to define the continuum in order to normalize the individual line profiles. A simple average over a few pixels on each side of the line is not accurate enough and can introduce undesirable uncertainties in the equivalent width measurements. We rely instead on a procedure similar to that outlined in LBH to define the location of the continuum in the far wings of the hydrogen Balmer lines.

We first construct a very high signal-to-noise template spectrum by combining our 27 spectroscopic observations of GD 323. This template spectrum displayed at the top of Figure 2 is then forced to match each single observation of GD 323 by multiplying the template by a polynomial of fifth degree in $\lambda$. The result of this procedure for a typical spectrum of GD 323 is illustrated in Figure 3. We define the continuum level over a given interval on the basis of the monochromatic flux in the template spectrum at the boundaries of the interval. We choose the following intervals over which the equivalent widths are to be measured: 4800-4925 $\AA, 4275-4425 \AA, 4065-4150 \AA$, corresponding to $\mathrm{H} \beta, \mathrm{H} \gamma$, and $\mathrm{H} \delta$, respectively, as well as 4430-4510 $\AA$ and $4680-4750 \AA$ for the He I $\lambda 4471$ and He I $\lambda 4713$ features, respectively. We concentrate on these features since they are the strongest lines in the spectrum; other lines are visible, but are either weak or blended and can only be measured with difficulty. Furthermore, the wavelength intervals are chosen to include as much of the line wings as possible, while avoiding blending. The intervals over which the equivalent widths are measured are reproduced in Figure 3.

The equivalent width measurements as a function of time for the three hydrogen and the two neutral helium absorption lines in GD 323 are displayed in Figure 4 for the 14 spectra secured within the single night of 2004 February 10. These results show clearly that the hydrogen and He I $\lambda 4471$ line strengths of GD 323 vary with time, and that these variations appear to be quasi-periodic. This was by no means expected, as we could very well have detected random variations, or no variations at all. Moreover, the observed variations occur on a relatively short timescale, with the feature strengths going from a maximum to a minimum over the course of a few hours only. Even more surprising, the variations of the hydrogen lines and $\lambda 4471$ appear to be out of phase: as the hydrogen lines (especially $\mathrm{H} \beta$ and $\mathrm{H} \gamma$ ) get stronger, the helium line weakens and vice versa. The case for He I $\lambda 4713$ is admittedly much weaker and we cannot, at this stage, account completely for its behavior: while the line is reasonably strong and unaffected by blending, the variations it displays 
appear more muted than those observed in $\lambda 4471$, and their phasing with the hydrogen lines much less obvious.

\section{2. $\quad$ Error Analysis}

A preliminary estimate of the errors in the equivalent width measurements for the three hydrogen lines in GD 323 has been obtained from a consideration of the spectrum of the comparison star PG 1234+482. We have measured the equivalent widths for each line on the 13 individual fluxed spectra of that star secured on 2004 February 10. The rms error on the equivalent widths reflects the $\mathrm{S} / \mathrm{N}$ ratio of the individual spectra and the reliability of the procedure used to set the continuum level (§ 2). They amount to $0.03 \AA$ for $\mathrm{H} \beta, 0.08$ $\AA$ for $\mathrm{H} \gamma$, and $0.03 \AA$ for $\mathrm{H} \delta$. These errors can be considered as lower limits, as the use of smoothed flux calibration functions applied to the raw individual spectra of PG $1234+482$ guarantees a perfect match of the continuum flux in individual spectra with the template of that star. When the same smoothed flux calibration functions are applied to the raw individual spectra of GD 323, the match to the average, or template, spectrum of GD 323 is not as good, since residual calibration errors remain. It is not possible to evaluate these calibration errors, however, as they cannot be disentangled from the intrinsic variations of GD 323.

To estimate these residual calibration errors, we have opted to go back to the 13 individual IRAF-reduced spectra of PG 1234+482 acquired on 2004 February 10. Because these spectra were simply reduced with the standard IRAF package, the rms deviations over a single night are deemed to include contributions from the $\mathrm{S} / \mathrm{N}$ ratio of the individual spectra, from the continuum setting and, more importantly, from any residual calibration error associated with IRAF. While our standard reduction is decoupled from $\operatorname{IRAF}(\S 2.2)$, it is our feeling that the rms errors generated in this way are more representative of the true uncertainties associated with our procedure. Their values are: $0.27 \AA$ for $\mathrm{H} \beta$, and $0.16 \AA$ for $\mathrm{H} \gamma$ and $\mathrm{H} \delta$.

These IRAF-based error bars are included in Figure 4. For the neutral helium lines $\lambda 4471$ and $\lambda 4713$, both absent in the spectrum of PG $1234+482$, we also measure the rms errors for $\mathrm{H} \epsilon(0.20 \AA)$, the Balmer line that is closest in average equivalent width to the two weaker He I lines. 


\subsection{Single-Night Variations in Calibrated Spectra}

An alternative way of illustrating these variations is to divide the template spectrum of GD 323 (shown in Fig. 3) by each observed spectrum. Were GD 323 a constant star, this procedure should yield a straight line with random noise. The results for 3 spectra obtained on 2004 February 10 are displayed in Figure 5 at carefully selected phases. The bottom spectrum corresponds to a phase where the hydrogen line equivalent widths go through a minimum in Figure 4, while the top one corresponds to a maximum, and the middle spectrum is for an intermediate value; the trend is in the opposite direction for the helium lines. Also shown are the wavelength intervals used to measure the equivalent widths. Our results indicate that the flux variations never exceed $5 \%$ throughout the spectrum. A close examination reveals, however, that in the case of the 1043 spectrum, there is less flux in the hydrogen features and more flux in the He I features, while the opposite is true for the 1226 spectrum, even when taking into account the noise level. By integrating these small variations over the entire line profiles, we reduce the effect of the noise, and increase the significance of these variations. Indeed, even a $1 \%$ flux variation over a $100 \AA$ wide $\mathrm{H} \beta$ line profile yields a $1 \AA$ equivalent width variation, which is roughly the size of the variations observed in Figure 4.

\subsection{Single-Night Variations in IRAF-calibrated and Uncalibrated Spectra}

To reinforce these conclusions, we have carried out in parallel an analysis of the spectroscopic observations of GD 323 and PG $1234+482$, but this time on data that were flux calibrated with IRAF. An additional analysis was also carried out directly with the unfluxed spectra. In both cases, the quasi-periodic variations in the hydrogen and helium features uncovered in $\S 3.1$ could also be detected, although the quality of the results was significantly

reduced. The fact that we were able to achieve similar results via several methods makes it unlikely that the variations uncovered in this paper are due to some artifice in the data acquisition or reduction procedures.

\subsection{Variations over longer timescales}

The equivalent width measurements for all five successive nights - a total of 27 observations - are shown in Figure 6 for the aforementioned features. The variations observed on the first night persist on the following nights, although only a segment of the quasi-sinusoidal variations is observed. Remarkably, on a given night, all hydrogen lines follow a similar pat- 
tern of variations, while the helium lines also share a common pattern, different from that of the hydrogen lines. Our attempts to fit a single periodic function through all the data points have failed, indicating that the variations are only quasi-periodic.

\section{DISCUSSION}

The spectroscopic variations uncovered in GD 323 occur on timescales of the order of $3.5 \mathrm{~h}$. Were these variations associated with the rotation of the underlying white dwarf $\left(R=1.4 \times 10^{-2} R_{\odot}\right)$, they would correspond to an equatorial velocity $v=5 \mathrm{~km} \mathrm{~s}^{-1}$. This number is quite in line with the idea that most white dwarfs of both DA and DB types are very slow rotators (Heber et al. 1997b; Koester et al. 1998; Dufour et al. 2002; Karl et al. 2004). This suggests that the association of the $3.5 \mathrm{~h}$ timescale with stellar rotation is reasonable.

It is also possible to revisit the issue of secular spectroscopic variations on timescales of years in the spectrum of GD 323. The first digital optical spectra of GD 323 were obtained over 20 years ago, but the different instrumental setups and resolutions used always hampered a direct comparison with spectra secured more recently (Liebert et al. 1984; Koester et al. 1994). However, the setup for the observations of Koester et al. (1994) and ours is quite similar and this provides a better basis for the comparison of high $\mathrm{S} / \mathrm{N}$ ratio spectra secured 11 years apart. A comparison of the average spectrum of Koester et al. (1994) for the night of 1993 March 31 (average of 6 spectra) with our so-called template spectrum (average of 27 spectra) is shown in Figure 7. Although the reduction procedures differ, the claim can certainly be made that there are no large-amplitude secular variations in the spectrum of GD 323 over that baseline. However, small variations, most notably in He I $\lambda 4026$ and He I $\lambda 4471$, may be present.

The detection of spectroscopic variations on a $3.5 \mathrm{~h}$ timescale in GD 323 forces us to revise our ideas about this unusual object. As discussed in the Introduction, the thorough analysis of Koester et al. (1994) suggested that none of the options they considered provided a completely satisfactory fit to the data, but the stratified model provided the most acceptable match to GD 323. It seems difficult to understand now how the spectroscopic variations uncovered here could be accommodated by a static, layered model or, for that matter, by a static homogeneous hydrogen-rich atmosphere containing traces of helium.

Our findings suggest instead that an inhomogeneous surface abundance distribution in a slowly rotating star should be favored. Models with inhomogeneous surface abundances were discussed by Beauchamp et al. (1993), who considered several belt and cap geometries, 
and by Koester et al. (1994), who considered a spot model. Can such models reproduce the basic characteristics of GD 323 ? Let us consider the conclusions of Koester et al. (1994) first: they model the spot geometry by adding appropriately weighted fluxes from model atmospheres of DA and DB stars and restrict, on physical grounds, the difference in effective temperatures of these models to less than $5000 \mathrm{~K}$. The conclusion they derive from the analysis of spotted models is a familiar one (see, e.g., Liebert et al. 1984); namely that it is difficult to find a single model (characterized here by the effective temperature of the hydrogen-rich and helium-rich spots and by the relative surface they cover) that matches both the hydrogen and helium line strength and the slope of the energy distribution of GD 323.

In an earlier analysis, Beauchamp et al. (1993) had used more sophisticated models that considered specific geometries (equatorial belts and polar caps) and included a selfconsistent treatment of the limb darkening effects. Sample optical spectra of models with a pure helium equatorial band and pure hydrogen polar caps from that investigation are reproduced here in Figure 8 and contrasted with our average spectrum of GD 323. These results illustrate how the strength of the hydrogen and helium lines, and in particular their relative strength, depends on both the extent of the helium-rich belt and on the inclination of the stellar symmetry axis with respect to the line of sight (given here by the angle $\theta_{\text {axis }}$ ). For a given half-width of the pure helium equatorial band of $\Delta \alpha=45^{\circ}$, the hydrogen lines are stronger and the helium lines weaker when the star is seen pole-on $\left(\theta_{\text {axis }}=0^{\circ}\right)$ instead of equator-on $\left(\theta_{\text {axis }}=90^{\circ}\right)$. Similarly, for a given inclination axis of $\theta_{\text {axis }}=0^{\circ}$ (pole-on), the helium lines become stronger and the hydrogen lines weaker when the half-width of the pure helium equatorial band is increased from $\Delta \alpha=45^{\circ}$ to $60^{\circ}$. In the present context, variations of the hydrogen and helium line strengths in opposite phase could be reproduced if the rotation axis and the symmetry axis are misaligned. While no optimal fit is provided here or in the investigation of Beauchamp et al. (1993), the results of Beauchamp et al. appear more encouraging than those of Koester et al. (1994). In particular, they encountered no major difficulty in matching the energy distribution with a model that gave an acceptable, although admittedly far from perfect, fit to the average optical spectrum of GD 323.

The modeling of surface inhomogeneities in terms of spots, or caps and belts, can perhaps lead one to believe that the origin of these surface features must be directly related to the presence of a magnetic field at the stellar surface. In that picture, GD 323 would be a low-field analog of Feige 7, a DAB white dwarf with a $35 \mathrm{MG}$ dipolar field (Achilleos et al. 1992). In GD 323, the null circular polarization measurement of Angel et al. (1981) yields a $3 \sigma$ upper limit of $3 \mathrm{MG}$ on the longitudinal field component at the stellar surface. However, the idea that surface inhomogeneities are related to other processes, such as the convective dilution of a thin overlying hydrogen envelope, should also be entertained. Indeed, it has 
been pointed out a while back (Liebert et al. 1987) that GD 323 is slightly cooler than the red edge of the DB gap, that peculiar region between 45,000 K and 30,000 K that appears to be devoid of helium-atmosphere degenerates. Models of the spectral evolution of white dwarfs (e.g., Fontaine \& Wesemael 1987) attempt to account for this gap by calling on the dilution of a thin hydrogen layer at the surface of a 30,000 K DA star with the more massive helium envelope, dilution that would lead to a $\mathrm{DA} \rightarrow \mathrm{DB}$ transition near that effective temperature. If GD 323 were understood in those terms, an idea that we favor, its surface might be characterized by the presence of horizontal abundance gradients. Unfortunately, it is not possible to be more specific since a complete picture of the mixing process still eludes us at this stage. The geometries considered in the exploratory investigations of Beauchamp et al. (1993) and Koester et al. (1994) appear reasonable starting points for a renewed and more thorough study of that possibility.

We thank the director and staff of the Steward Observatory for the use of their facilities, and A. Beauchamp for his essential contribution to the calculation of models with surface inhomogeneities. This work was supported in part by the NSERC Canada and by the Fund FQRNT (Québec). 


\section{REFERENCES}

Achilleos, N., Wickramasinghe, D. T., Liebert, J., Saffer, R. A., \& Grauer, A. D. 1992, ApJ, 396,273

Angel, J. R. P., Borra, E. F., \& Landstreet, J. D. 1981, ApJS, 45, 457

Beauchamp, A., Wesemael, F., Fontaine, G., \& Bergeron, P. 1993, in White Dwarfs: Advances in Observation and Theory, ed. M. A. Barstow, NATO ASI Series, vol. 403, 281

Bergeron, P., \& Liebert, J. 2002, ApJ, 566, 1091

Burleigh, M., Bannister, N., \& Barstow, M. 2001, in ASP Conf. Ser. 226, 12th European Workshop on White Dwarf Stars, ed. J. L. Provencal, H. L. Shipman, J. MacDonald, \& S. Goodchild (San Francisco: ASP), 135

Dufour, P., Wesemael, F., \& Bergeron, P. 2002, ApJ, 575, 1025

Fontaine, G., \& Wesemael, F. 1987, in I.A.U. Colloquium 95, The Second Conference on Faint Blue Stars, ed. A.G.D. Philip, D.S. Hayes, \& J. Liebert (Schenectady: L. Davis Press), 319

Heber, U., Napiwotzki, R., Lemke, M., \& Edelmann, H. 1997a, A\&A, 324, L53

Heber, U., Napiwotzki, R., \& Reid, I. N. 1997b, A\&A, 323, 819

Holberg, J. B., Kidder, K. M., \& Wesemael, F. 1990, ApJ, 365, L77

Jordan, S., Heber, U., Engels, D., \& Koester, D. 1993, A\&A, 273, L27

Karl, C., Napiwotzki, R., Heber, U., Dreizler, S., Koester, D., \& Reid, I. N. 2004, A\&A, in press

Kidder, K. M., Holberg, J. B., Barstow, M. A., Tweedy, R. W., \& Wesemael, F. 1992, ApJ, 394,288

Koester, D. 1991a, in Evolution of Stars: The Photospheric Abundance Connection, ed. G. Michaud \& A. Tutukov, (Dordrecht: Kluwer), 435

Koester, D. 1991b, in White Dwarfs, ed. G. Vauclair \& E.M. Sion, NATO ASI Series, Series C, no. 336 (Dordrecht: Kluwer), 343

Koester, D., Dreizler, S., Weidemann, V., \& Allard, N. F. 1998, A\&A, 338, 612 
Koester, D., Liebert, J., \& Saffer, R. A. 1994, ApJ, 422, 783

Liebert, J., Bergeron, P., \& Holberg, J. B. 2005, ApJS, 156, 47 (LBH)

Liebert, J., Fontaine, G., \& Wesemael, F. 1987, Mem. Soc. Astr. Italiana, 58, 17

Liebert, J., Wesemael, F., Sion, E. M., \& Wegner, G. 1984, ApJ, 277, 692

Maxted, P. F. L., Burleigh, M. R., Marsh, T. R., \& Bannister, N. P. 2002, MNRAS, 334, 833

McCook, G. P., \& Sion, E. M. 1999, ApJS, 121, 1

Napiwotzki, R. and the SPY Consortium 2005, in ASP Conf. Ser., 14th European Workshop on White Dwarfs, ed. D. Koester \& S. Moehler, in press

Oke, J. B., Weidemann, V., \& Koester, D. 1984, ApJ, 281, 276

Robinson, E. L., \& Winget, D. E. 1983, PASP, 95, 386

Vennes, S., Dupuis, J., \& Chayer, P. 2004, ApJ, 611, 1091

Wesemael, F., Bergeron, P., \& Briand, E. 1995, JRASC, 89, 183

Wesemael, F., Bergeron, P., Lamontagne, R., Fontaine, G., Beauchamp, A., Demers, S., Irwin, M. J., Holberg, J. B., Kepler, S. O., \& Vennes, S. 1994, ApJ, 429, 369

Wesemael, F., Greenstein, J. L., Liebert, J., Lamontagne, R., Fontaine, G., Bergeron, P., \& Glaspey, J. W. 1993, PASP, 105, 761

Wolff, B., Jordan, S., Koester, D., \& Reimers, D. 2000, A\&A, 361, 629 
Fig. 1.- Optical spectra of GD 323 (left panel) and PG 1234+482 (right panel) taken on 2004 February 10. The spectra appear in chronological order from the bottom up and are labeled with the corresponding universal time at which the integration was started. The spectra have been flux calibrated, normalized to unity at $4600 \AA$ and offset from each other by a factor of 0.25 for clarity.

Fig. 2.- Optical spectra of GD 323 (left panel) and PG 1234+482 (right panel) taken on 2004 February 11-14. The spectra appear in chronological order from the bottom up and are labeled with the corresponding universal time at which the integration was started. The spectra have been flux calibrated, normalized to unity at $4600 \AA$ and offset from each other by a factor of 0.25 within the same night and by 0.3 between consecutive nights. The combined spectrum of GD 323 from all nights is also displayed at the top of the left panel; for comparison, the combined spectrum of PG $1234+482$ is displayed at the top of the right panel.

Fig. 3.- Template spectrum (average of 27 spectra) superimposed on a typical spectrum of GD 323. Here, the template continuum has been adjusted to that of the observed spectrum using the procedure described in the text. This is done for each individual spectrum. The wavelength range used to measure the equivalent widths is shown for the hydrogen (dotted lines) and the He I (dashed lines) absorption lines.

Fig. 4.- Equivalent widths as a function of time (hours UT) for the spectra of GD 323 taken on 2004 February 10. The panels correspond to $\mathrm{H} \beta, \mathrm{H} \gamma, \mathrm{H} \delta$, He I $\lambda 4471$ and He I $\lambda 4713$ from top to bottom. The error bars are estimated from the equivalent width measurements of PG $1234+482$, which showed no significant variations and had a similar signal-to-noise ratio. These values are then used as error bars for GD 323.

Fig. 5.- Results obtained by dividing the fitted template spectrum of GD 323 (see, e.g., Fig. 3) by the observed spectra. The spectra are all taken on 2004 February 10, and they are labeled with the universal time of their acquisition. The spectra selected correspond to various phases where the hydrogen line strengths go from a minimum to a maximum, from bottom to top. The wavelength range used for the equivalent width measurements are indicated for the five strong features: $\mathrm{H} \beta-\mathrm{H} \delta$ (dotted lines), He I $\lambda 4471$ and He I $\lambda 4713$ (dashed lines).

Fig. 6. - Equivalent widths as a function of relative time (in hours) for the spectra of GD 323 taken over five nights, namely from 2004 February 10-14. The first observation is offset from $t=0$ by one hour. The panels correspond to $\mathrm{H} \beta, \mathrm{H} \gamma, \mathrm{H} \delta$, He I $\lambda 4471$ and He I $\lambda 4713$ from top to bottom. The error bars are the same as those shown in Figure 5 but are omitted for clarity. 
Fig. 7.- Comparison of our template spectrum of GD 323 (average of 27 spectra) with that generated from the data of Koester et al. (1994) for the night of 1993 March 31 (average of 6 spectra). The spectra are normalized to unity at $4600 \AA$ and offset from each other by a factor of 0.2 . The instrumental setup was similar between the two sets of observations, but the reduction procedure was different (see text).

Fig. 8. - Sample optical spectra of models with a pure helium equatorial band and pure hydrogen polar caps; the top spectrum is that of GD 323 (average of 27 observations). The spectra are normalized to unity at $4250 \AA$ and offset from each other by a factor of 0.35 . The half-width of the pure helium equatorial band is given by $\Delta \alpha$, while $\theta_{\text {axis }}$ represents the angle between the stellar symmetry axis and the line of sight. 


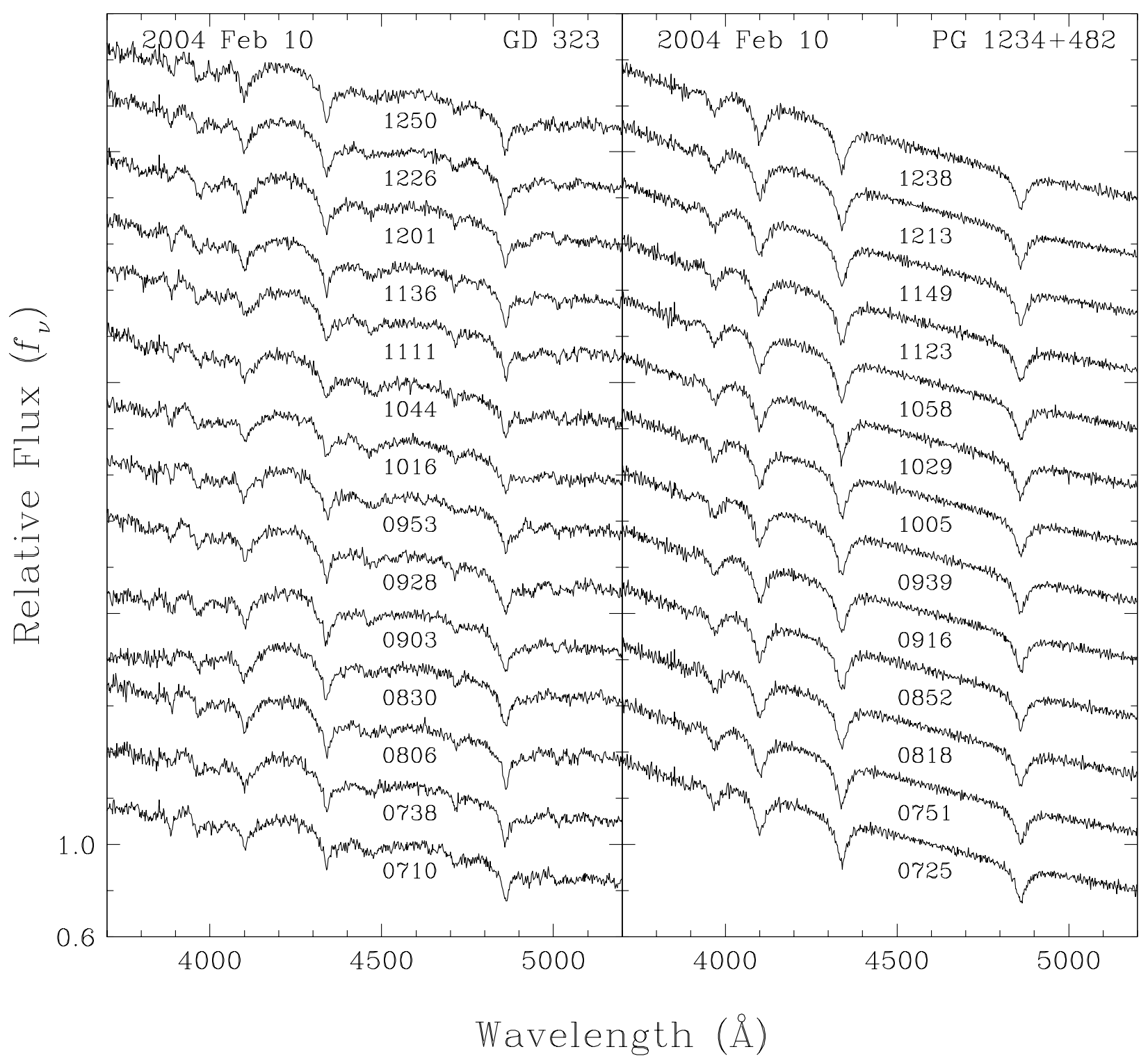

Figure 1 


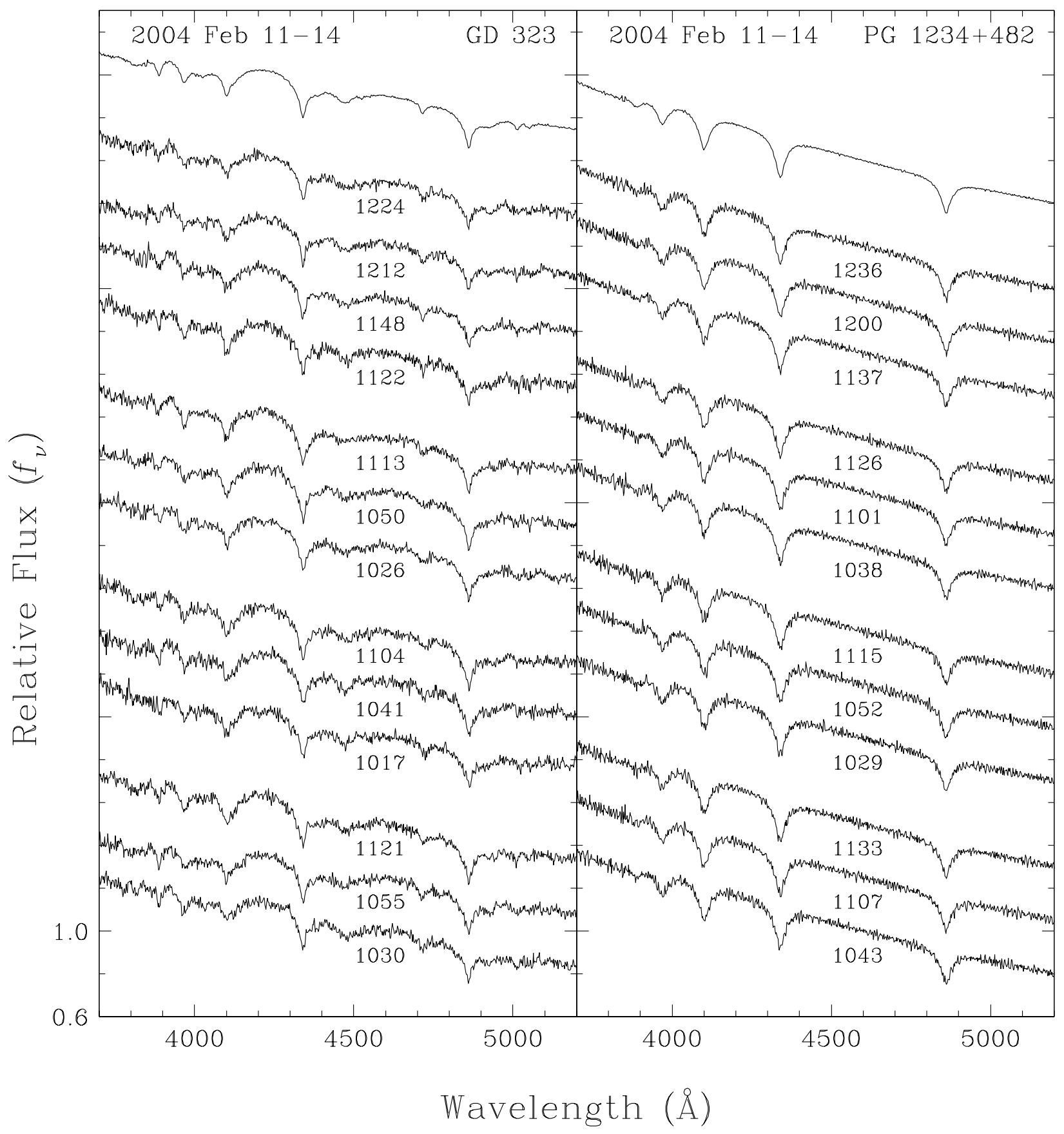

Figure 2 


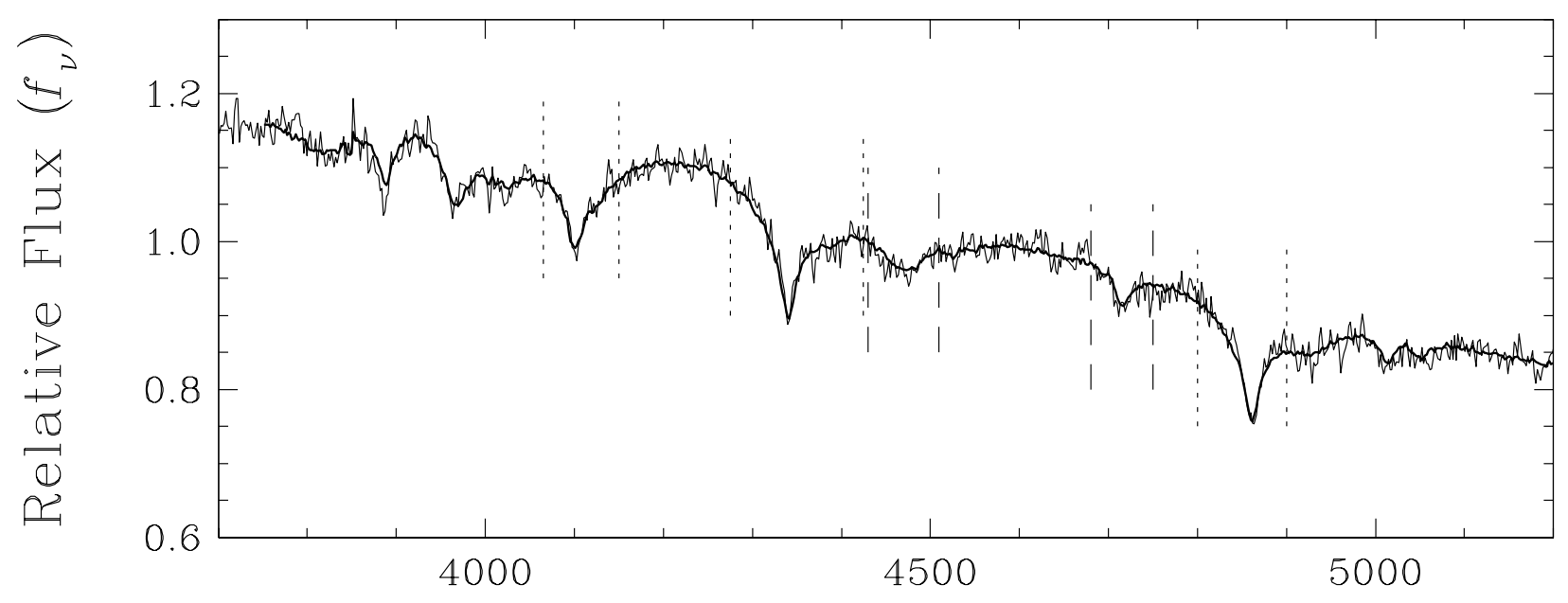

Wavelength $(\AA)$

Figure 3 


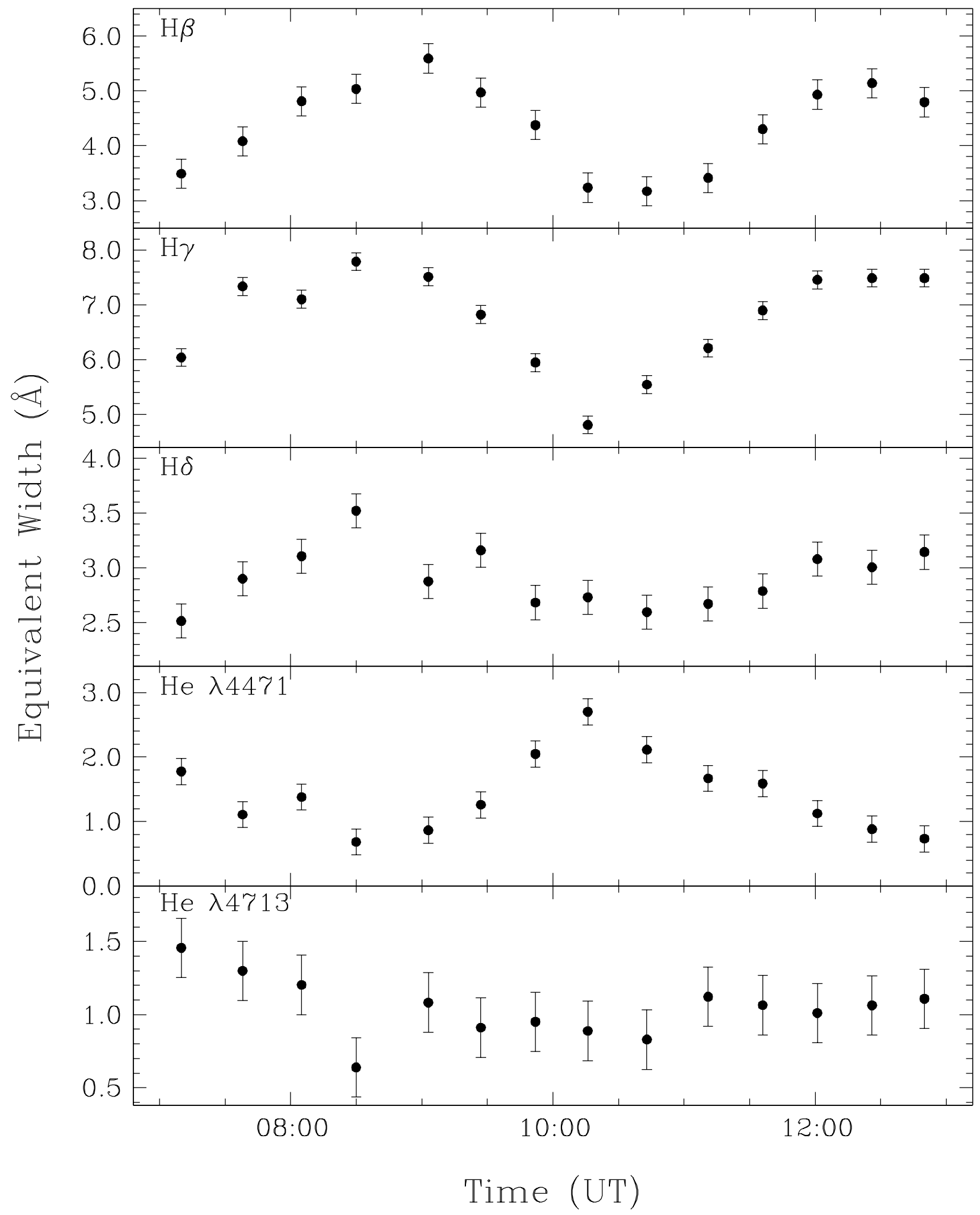

Figure 4 


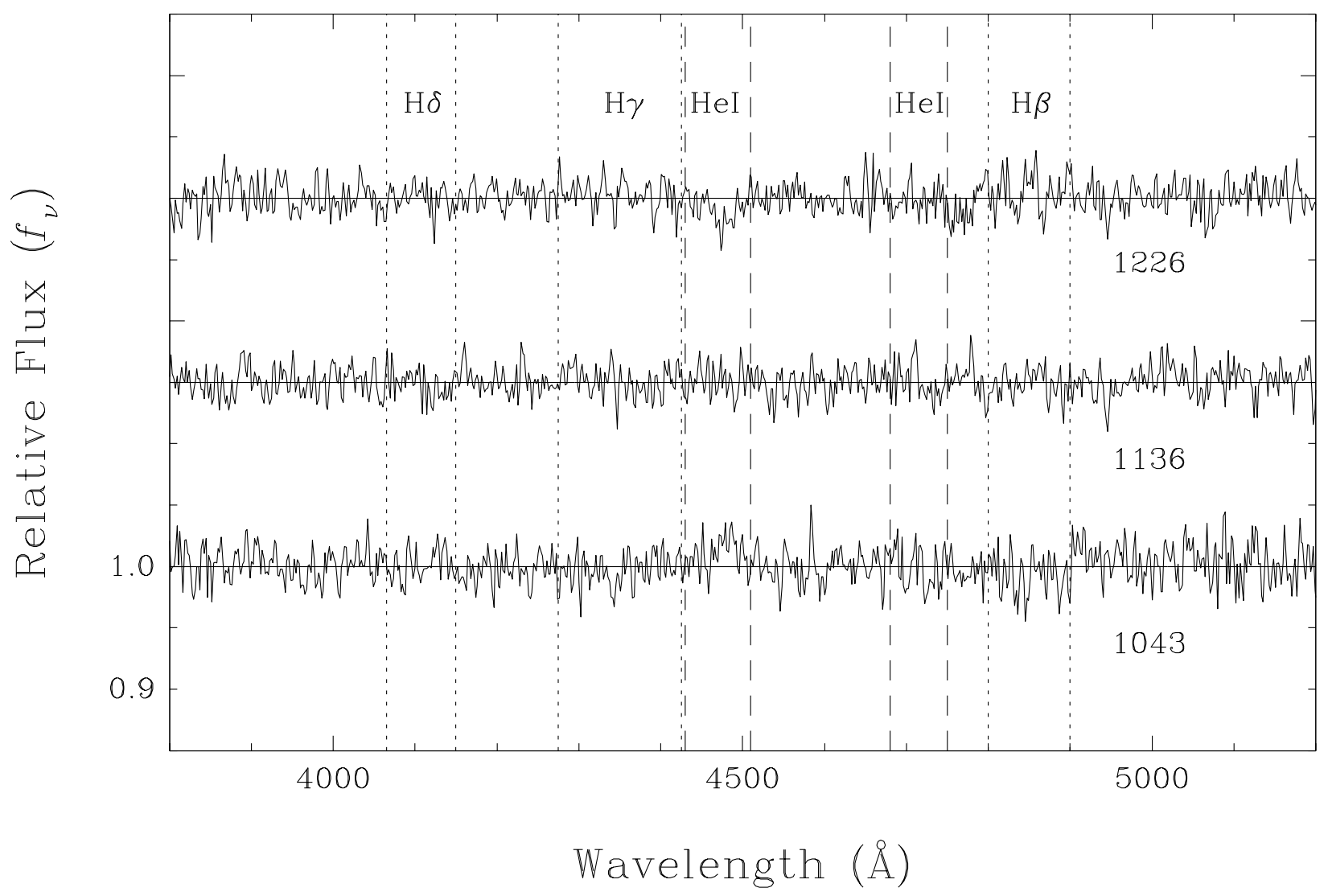

Figure 5 


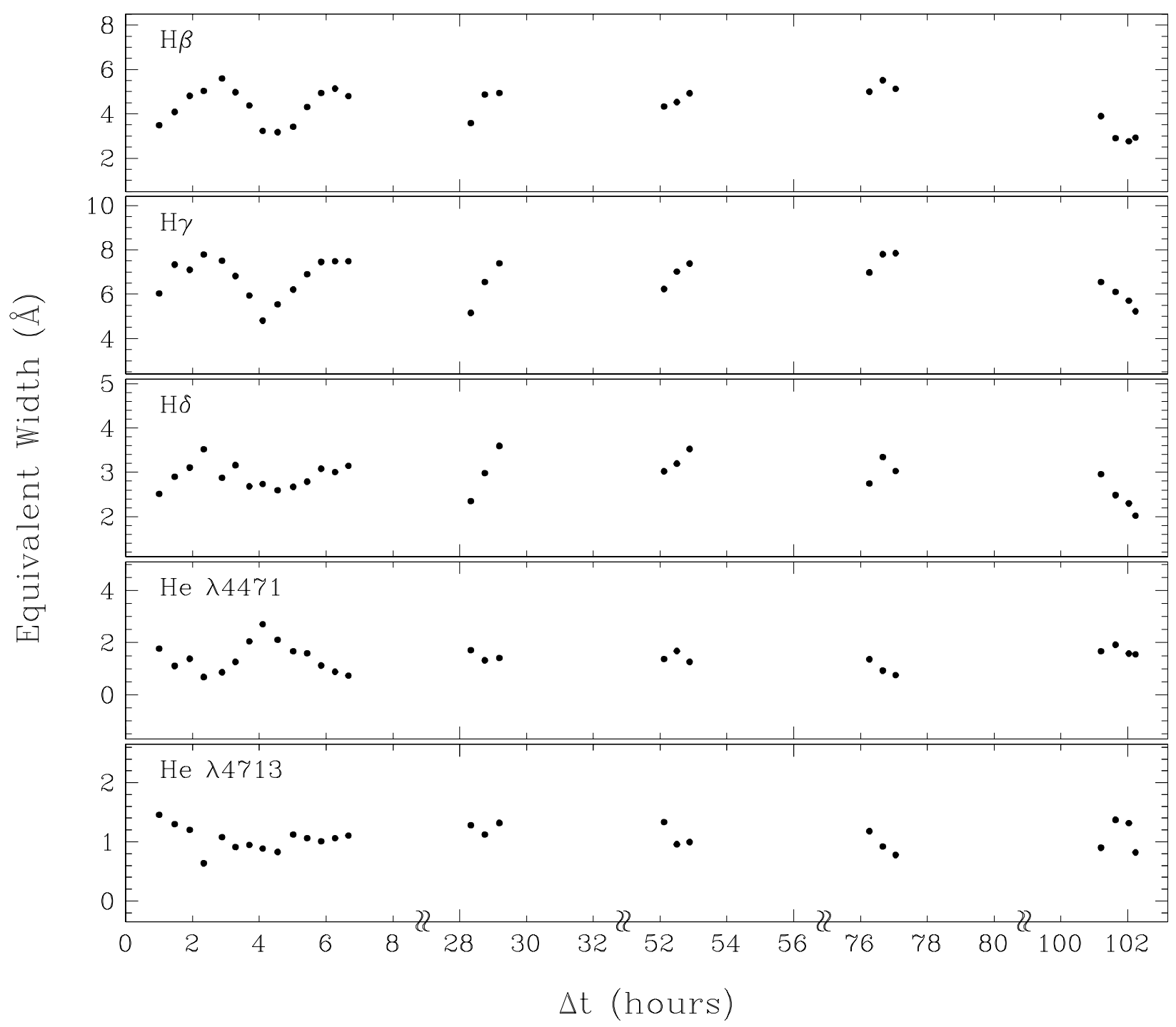

Figure 6 


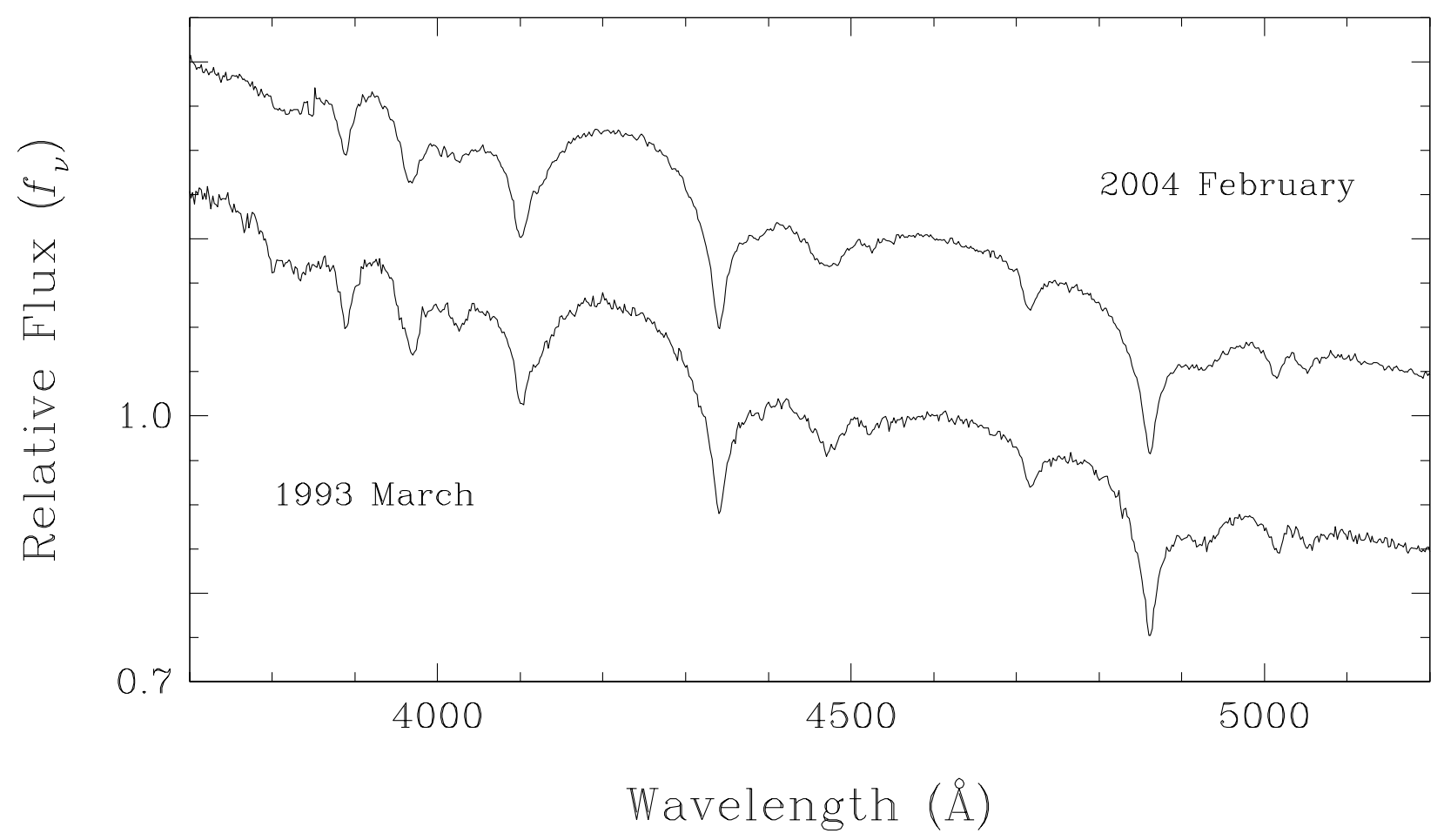

Figure 7 


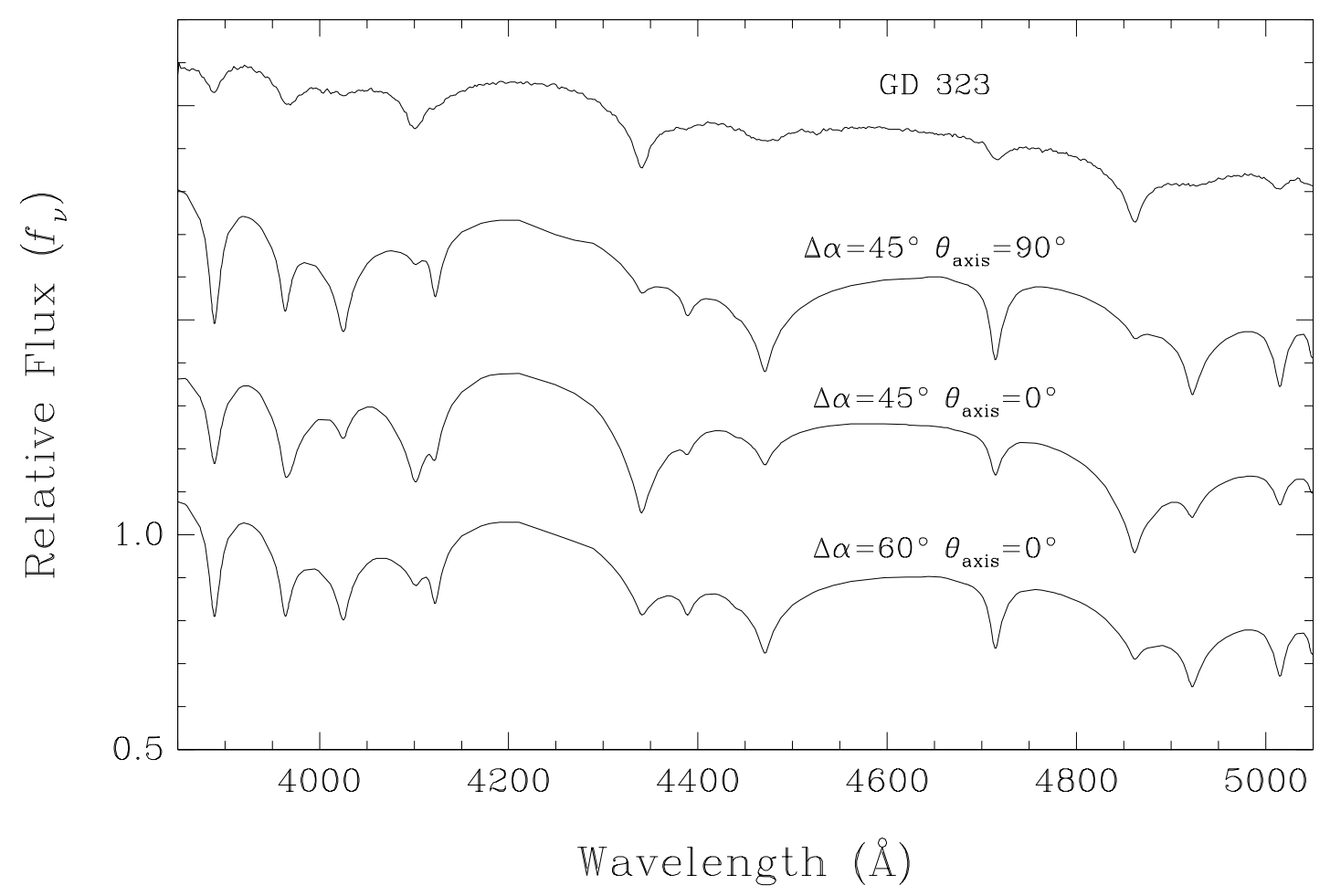

Figure 8 